\title{
THE MAGNITUDE OF THE EXTRACELLULAR SPACE IN ELECTRON MICROGRAPHS OF SUPERFICIAL AND DEEP REGIONS OF THE CEREBRAL CORTEX
}

\author{
A. VAN HARREVELD AND JANA STEINER \\ California Institute of Technology, Division of Biology, I20I East California \\ Boulevard, Pasadena, California gIIO9, U.S.A.
}

S U M M A R Y

Electron micrographs (EMs) of central nervous tissue perfused with glutaraldehyde and post-fixed with osmium tetroxide exhibit more extracellular space in superficial than in deep regions of the brain. It was postulated that this is due to the availability to the surface tissue of the cerebrospinal fluid in the subarachnoidal space. It was assumed that in addition to the extracellular material this fluid is taken up by dendritic elements in the surface layer of the cortex during the glutaraldehyde perfusion. Indeed, dendritic structures in EMs of the surface layer of glutaraldehyde-perfused and $\mathrm{OsO}_{4}$-post-fixed cortex exhibited a more pronounced swelling than similar structures in deeper cortical regions, where only extracellular material was available to the dendrites. The extracellular space in such preparations consisted of narrow slits surrounding the tissue elements. These slits were formed during the post-fixation since they were not present in freeze-substituted, glutaraldehyde-perfused tissue, which showed a paucity of extracellular material. The slits were in general wider in the superficial than in the deep cortical tissue. That this difference is related to the amount of material taken up by the dendrites during the glutaraldehyde perfusion was supported by experiments in which the exposed cortical surface was flooded with Ringer's solution during the glutaraldehyde perfusion. This resulted in a ballooning of dendritic elements in the cortical surface due to the availability of an unlimited amount of fluid on the cortex. Post-fixed material of these flooded cortices yielded EMs with unusually wide extracellular slits around the tissue elements.

\section{INTRODUCTION}

In electron micrographs (EMs) of central nervous tissue fixed by glutaraldehyde perfusion and osmium-tetroxide post-fixation more extracellular space was observed in the superficial than in the deeper regions of the brain (Karlsson \& Schultz, 1965; Johnston \& Roots, 1967). Because of the large changes in extracellular space which have been found to occur during perfusion with glutaraldehyde and $\mathrm{OsO}_{4}$ post-fixation (Van Harreveld \& Khattab, I968; Van Harreveld \& Steiner, 1970) and probably during embedding (Nevis \& Collins, 1967) the space in EMs of such tissues cannot be directly equated with the extracellular space in the living material, and the possibility has to be considered that differences in space observed in superficial and deep central nervous tissue are produced during the fixation and embedding procedure.

With the freeze-substitution technique, which can be expected to preserve the water distribution in central nervous tissue more faithfully than conventional fixation (Van 
Harreveld, Crowell \& Malhotra, 1965), it has been shown that during glutaraldehyde perfusion there is a movement of extracellular material into certain cellular elements which become swollen and electron-transparent (Van Harreveld \& Khattab, I968; Van Harreveld \& Steiner, 1970). It has been shown, furthermore, that when the cortex is flooded with Ringer's solution during the perfusion with glutaraldehyde the swelling of the cortical elements is greatly enhanced. This enhanced swelling is restricted to the surface layers of the cortex and it was concluded that under these conditions superficial cellular elements can take up not only the extracellular material, but also some of the fluid flooding the cortex (Van Harreveld \& Khattab, 1968).

Even when the cortex is not intentionally flooded the superficial layers have access to the thin layer of cerebrospinal fluid in the subarachnoidal space, and it seems likely that during perfusion fixation this fluid is taken up by the same cellular elements that absorb the extracellular material. One can therefore expect a more pronounced swelling of these structures in the surface than in the deeper layers of the cortex, where only the extracellular material is available.

During the $\mathrm{OsO}_{4}$ post-fixation of glutaraldehyde-perfused cortex an additional redistribution of water occurs in the tissue. In material prepared by glutaraldehyde perfusion followed by freeze substitution the tissue elements are closely approximated and 5-layered junctions between adjoining cell membranes are common. The $\mathrm{OsO}_{4}$ post-fixation prior to freezing results in the formation of more or less uniformly wide extracellular slits which tend to surround the tissue elements (Van Harreveld \& Khattab, 1968; Van Harreveld \& Steiner, 1970). In the cerebellum this is accompanied by a shrinking of tissue elements (fibres of Bergmann) which have taken up extracellular material during the glutaraldehyde perfusion (Van Harreveld \& Steiner, I970). It would seem possible that the formation of extracellular slits during post-fixation is affected quantitatively by the degree of swelling of elements during the glutaraldehyde perfusion. In this way a difference in the extracellular space in surface and deeper layers of the conventionally glutaraldehyde- $\mathrm{OsO}_{4}$ fixed tissue could be explained. In the present paper experiments designed to test the above postulate are described.

\section{METHODS}

The brains of white mice narcotized with urethane were fixed by perfusion with a glutaraldehyde solution through the abdominal aorta as described previously (Van Harreveld \& Khattab, I 968). The perfusion was carried out at a pressure of $13 \mathrm{~cm}$ of mercury ( $1725 \mathrm{kN} \mathrm{m}^{-2}$ ) first for Io s with Ringer's solution followed by the fixative warmed to $38^{\circ} \mathrm{C}$ for $10 \mathrm{~min}$. The fixative consisted of a $2.5 \%$ glutaraldehyde solution in a $0.067 \mathrm{M}$ phosphate buffer at $\mathrm{pH} 7.4$ with $0.5 \% \mathrm{NaCl}$ added. The osmolarity of the fixative determined cryoscopically was $640 \mathrm{~m}$-osmol. To prevent a pressure build-up in the brain case the calvarium and dura were removed over one hemisphere as described previously (Van Harreveld \& Malhotra, i 967). After the perfusion cortical tissue was isolated from the unexposed side and post-fixed for $2 \mathrm{~h}$ in $\mathrm{I} \% \mathrm{OsO}_{4}$ solution in the phosphate buffer at $4{ }^{\circ} \mathrm{C}$. In some experiments the exposed cortex was flooded during the perfusion with Ringer's solution warmed to $38^{\circ} \mathrm{C}$, after which it was similarly post-fixed. The tissue blocks were dehydrated in acetone, passed through propylene oxide and embedded in Maraglas (Freeman \& Spurlock, 1962). Thick ( $1 \mu \mathrm{m}$ ) sections stained with methylene blue and azure II (Richardson, Jarett \& Finke, I960) were examined with the light microscope. Thin sections treated with lead citrate (Reynolds, 1963) were used for electron microscopy. 
In other experiments square pieces of the exposed and unexposed cortex were placed in troughs of aluminium foil and subjected to freeze substitution as described previously (Van Harreveld \& Crowell, 1964). Acetone (containing $2 \% \mathrm{OsO}_{4}$ ) was used for the substitution. After substitution the tissue was embedded as described above.

\section{RESULTS}

\section{A comparison of superficial and deep cortical tissue from the unexposed hemisphere}

Figure I shows a low-magnification EM from the surface of the cortex perfused with glutaraldehyde and post-fixed with $\mathrm{OsO}_{4}$. The pia and the glial limiting membrane characterized by glycogen granules are present. The electron density of the elements in the underlying neuropil varies widely. Electron-transparent elements, which often can be identified by synaptic contacts as dendritic, contrast with more electron-dense structures, many of which can be characterized as presynaptic terminals by synaptic vesicles, or as glia containing glycogen granules. The figure shows a large and a smaller dendrite which contain neurotubules. Smaller electron-transparent structures may be dendritic spines; many of them form typical synapses with axonal endings. Many of the swollen structures contain enlarged endoplasmic reticulum. Small profiles often present in groups may be cross-sections of non-myelinated axons. The EM is very similar to micrographs of similarly fixed superficial cortical tissue presented previously (Van Harreveld \& Khattab, I 968). A comparison of this material with EMs of non-asphyxiated, freeze-substituted cortex (Van Harreveld \& Malhotra, 1967) suggests that the dendritic structures have become enlarged and electrontransparent by the uptake of extracellular material and probably of cerebrospinal fluid out of the subarachnoidal space. As discussed previously, this postulate is supported strongly by the large increase in the electrical impedance of the cortical tissue during glutaraldehyde perfusion (Van Harreveld \& Khattab, I968).

Figure 2 shows a higher magnification EM of the same tissue. Extracellular spaces in the form of narrow slits surround the tissue elements. The slits usually have a width of IO-1 $5 \mathrm{~nm}$. They are especially prominent in areas where many small profiles (nonmyelinated fibres) are present.

Figure 3 shows a low-magnification EM of deep cortical tissue fixed by perfusion with glutaraldehyde and $\mathrm{OsO}_{4}$ post-fixation. Dendritic and presynaptic structures can be recognized by the criteria given above. The electron density of the cellular elements is more uniform than in the surface layer. The dendrites and the spines are still more electron-transparent than the presynaptic terminals, but the difference is less striking. The neurotubules in the dendrites are closer together than in the dendrites in Fig. I. Also the spines are smaller in the deeper than in the superficial layer of the cortex. All these differences are consistent with the postulate that the swelling of the dendritic elements in the surface layer is greater due to the uptake of cerebrospinal fluid from the subarachnoidal space, in addition to that taken up from the extracellular fluid. Figure 4 shows a higher magnification of the same material. The slits between the tissue elements are narrower and at some places 5 -layered junctions between the plasma membranes are formed (arrows) as described by Karlsson \& Schultz (1964, 1965). One can find in the EMs of the deeper cortical layers areas where the slits 
between the tissue elements are wider than those shown in Fig. 4. However, in accordance with the observations of Karlsson \& Schultz (1965) and Johnston \& Roots (I968), there seems to be less extracellular space in the EMs of the deeper regions of the cortex than in the superficial ones.

A comparison of superficial and deep cortical regions from the exposed hemisphere flooded with Ringer's solution during fixation

If the differences in extracellular spaces between the superficial and deep regions of the cortex described above were due to the availability of cerebrospinal fluid to the superficial region then it might be possible to produce still wider extracellular slits in post-fixed tissue by increasing the amount of fluid available for swelling by flooding the cortex with Ringer's solution during the perfusion with glutaraldehyde. As described previously (Van Harreveld \& Khattab, 1968) this results in an enormous swelling of dendritic structures in the superficial cortical region. Figure 5 shows such material at low magnification. The large electron-transparent structures in this EM can in several instances be identified as dendritic by synaptic contacts. They often contain grossly swollen endoplasmic reticulum. Extracellular slits are present between the tissue elements, which in many instances were considerably wider than those in the surface layer of the unexposed cortex. The higher magnification of this material shown in Fig. 6 features slits more than $30 \mathrm{~nm}$ wide. Electron micrographs of the deeper layers of the flooded cortex resemble those of the deep tissue on the unexposed side, although they perhaps exhibit somewhat more extracellular space.

\section{Freeze-substituted cortical tissue}

The fluid shifts during glutaraldehyde perfusion and $\mathrm{OsO}_{4}$ post-fixation have been investigated with the method of freeze substitution which can be applied only to superficial tissue (Van Harreveld \& Khattab, 1968; Van Harreveld \& Steiner, 1970). These studies showed that during the perfusion extracellular material is taken up by cellular elements, resulting in a paucity of extracellular space, whereas the postfixation results in the creation of new spaces in the form of the narrow slits typical for $\mathrm{OsO}_{4}$-treated material. Figures 7 and 8 show the surface of unexposed cortex frozen after the glutaraldehyde perfusion. Figure 7 resembles Fig. I in the presence of enlarged, electron-transparent dendritic spines. However, the higher-magnification EM of this material (Fig. 8) shows little extracellular space. At many places the plasma membranes of adjoining elements are so closely approximated that they form 5layered junctions (arrows). Electron micrographs of unexposed cortex subjected to freeze substitution after perfusion and $\mathrm{OsO}_{4}$ post-fixation showed the more or less uniform slits surrounding the tissue elements as observed in the EM of the material similarly chemically fixed but not freeze-substituted (Fig. 2). These findings confirm the previous observations on the fluid shifts during glutaraldehyde perfusion and $\mathrm{OsO}_{4}$ post-fixation (Van Harreveld \& Khattab, I968; Van Harreveld \& Steiner, I 970).

The exposed and flooded cortex was also subjected to freeze substitution after glutaraldehyde perfusion, either with or without post-fixation. Figures 9 and 10 are EMs of this material frozen after the perfusion. The low magnification (Fig. 9) ex- 
hibits the greatly swollen postsynaptic structures also present in the conventionally prepared material shown in Fig. 5. The high magnification (Fig. I0) again shows relatively little extracellular space with close approximation of the plasma membranes to form 5-layered junctions (arrows) at several places. There is more extracellular space in these EMs than in similarly prepared tissue on the unexposed, non-flooded side (Fig. 8), however. Electron micrographs of the exposed and flooded cortex frozen after post-fixation have yielded results similar to those of the conventionally treated tissue shown in Fig. 6. They showed the relatively wide extracellular slits which characterize the latter EM. In the flooded tissue the wide extracellular slits also seem thus to be formed during the post-fixation.

In all the above experiments the tissue was perfused with glutaraldehyde for $10 \mathrm{~min}$. This resulted in a firm tissue which seemed well fixed and from which blocks could easily be cut. Many investigators have fixed tissue in glutaraldehyde for longer periods. In some of the present experiments cortical tissue was perfused for ro min, then placed in the glutaraldehyde solution for $2 \mathrm{~h}$ and subsequently freeze-substituted. When this procedure was carried out with unexposed cortex the resulting EMs showed tissue with somewhat more extracellular space than cortical tissue frozen immediately after the 10-min perfusion. However, when flooded cortex was used a very abundant extracellular space was found (Figs. I I, I2). The distribution of this space was quite different from that of tissue which had been post-fixed for the same period of time $\left(2 \mathrm{~h}\right.$ in the $\mathrm{OsO}_{4}$ solution). The latter material showed in both freeze-substituted and conventionally prepared tissues relatively wide slits surrounding the tissue elements. In tissue kept for $2 \mathrm{~h}$ in the glutaraldehyde solution the small tissue elements exhibited generally rounded contours with irregular rather than slit spaces between adjacent elements (Fig. 12): When exposed cortex, flooded during the perfusion and kept for $2 \mathrm{~h}$ in the glutaraldehyde solution, was post-fixed with $\mathrm{OsO}_{4}$ the distribution of the extracellular space tended to resemble that of cortical tissue post-fixed immediately after the 10-min perfusion (Fig. 6).

\section{DISCUSSION}

The results of this investigation support the postulate that the differences in extracellular space in EMs from superficial and deep areas of the cortex can be explained by the availability to the surface layers of a limited amount of cerebrospinal fluid in the subarachnoidal space. There is convincing evidence that dendritic structures can take up fluid bathing the cortical surface in addition to extracellular material (Van Harreveld \& Malhotra, 1967; Van Harreveld \& Khattab, 1968). This explains the more pronounced swelling of dendritic spines in the surface layers of the unexposed cortex as compared with the deep tissue. In cortex which is flooded during fixation the almost unlimited amount of fluid present accounts for the enormous dendritic swelling. It would seem that only the fluid bathing the cortex is available to the dendrites and that the perfusion fluid cannot serve this purpose. The dendritic swelling has been found to depend on the amount of extracellular and surface fluid available to the deep and superficial regions of the unexposed and exposed cortex to which the perfusion fluid 
can be expected to be available in the same amount. It would seem possible that the high osmolarity of the glutaraldehyde solution used for perfusion prevents its being taken up by the dendrites.

The width of the extracellular slits in glutaraldehyde-perfused and $\mathrm{OsO}_{4}$-post-fixed material seems to be correlated with the amount of fluid which is taken up by the dendritic structures during the glutaraldehyde perfusion. The slits were widest in cortex flooded during glutaraldehyde perfusion, less wide in the unexposed cortex, where the fluid in the subarachnoidal space is available for swelling, whereas in the deeper cortical layers where only extracellular fluid can move into the dendritic structures, the extracellular space in the post-fixed material was quite small. The formation of these slits occurs during the $\mathrm{OsO}_{4}$ post-fixation, perhaps by the creation of equal charges on opposing membranes (Van Harreveld \& Khattab, I968). There is evidence that in this process structures which take up fluid during the glutaraldehyde perfusion shrink again. The cerebellar glia (Bergmann fibres), which was enlarged and electron-transparent in EMs of material freeze-substituted after perfusion, was smaller and more electron-dense when the tissue was also post-fixed (Van Harreveld \& Steiner, 1970). The fluid which is released in this way may make up part or all of the extracellular material in the slits of post-fixed material. The larger swelling of dendritic elements in the surface layer of unexposed and especially of flooded cortex may yield during the $\mathrm{OsO}_{4}$ fixation more material for the creation of extracellular slits than in the deep cortical tissue.

It is of interest that EMs prepared by freeze substitution of cortical tissue which had been kept in the glutaraldehyde fixative for $2 \mathrm{~h}$ after I0-min perfusion fixation while being flooded with Ringer's solution exhibited large extracellular spaces. Since EMs of tissue flooded during the perfusion and frozen immediately afterwards showed a paucity of extracellular space it can be concluded that the large extracellular spaces were formed during the bathing of the tissue in the fixative. These large spaces were not observed in tissue of the unexposed side bathed for $2 \mathrm{~h}$ in the fixative. It can therefore be postulated that the extracellular material which was formed during the 2-h bath in the fixative was derived from the fluid in the grossly swollen dendrites in the flooded cortex and not from the glutaraldehyde solution which was available in equal abundance to both tissues.

The distribution of the extracellular space in EMs of flooded cortex bathed for $2 \mathrm{~h}$ after the perfusion in the glutaraldehyde solution is quite different from that of tissue post-fixed for an equally long time in $\mathrm{OsO}_{4}$. In the former irregularly shaped extracellular spaces are found between the small tissue profiles (non-myelinated axons), whereas in the post-fixed tissue the space consists of more or less uniformly wide slits surrounding the tissue elements, many of which have lost their rounded appearance. The space distribution in tissue long bathed in the fixative is very similar to that of cortical tissue perfused for 20 min with hydroxyadipaldehyde. This compound causes first a severe contraction of the extracellular space followed by an expansion (Van Harreveld \& Khattab, 1969). The action of glutaraldehyde seems to follow a similar course, although on a much more extended time base. It is of interest that $\mathrm{OsO}_{4}$ post-fixation tends to reduce the large extracellular spaces and to transform 
them into slits surrounding the tissue elements both in the hydroxyadipaldehydeperfused tissue and in the cortex bathed for $2 \mathrm{~h}$ in the glutaraldehyde fixative. This is a striking example of the powerful ability of $\mathrm{OsO}_{4}$ to shape the appearance of the tissue. The forces and reactions which cause the changes in water distribution in the tissue during the fixation procedure can be only guessed at. Whatever their nature they are of greater importance for the ultimate appearance of the tissue as seen under the electron microscope than the magnitude and distribution of the extracellular space in the living material.

This investigation was supported by a grant from the National Science Foundation (GB 6698) and from the Barber Fund.

\section{REFERENCES}

Freeman, J. A. \& Spurlock, B. O. (1962). A new epoxy embedment for electron microscopy. 7. Cell Biol. 13, 437-443.

Johnston, P. V. \& Roots, B. I. (1967). Fixation of the central nervous system by perfusion with aldehydes and its effect on the extracellular space as seen by electron microscopy. F. Cell Sci. 2, 377-386.

Karlsson, U. \& Schultz, R. (1964). Plasma membrane apposition in the central nervous system after aldehyde perfusion. Nature, Lond. 201, 1230-1231.

Karlsson, U. \& Schultz, R. L. (1965). Fixation of the central nervous system for electron microscopy by aldehyde perfusion. F. Ultrastruct. Res. 12, I60-1 86.

Nevis, A.H. \& Collins, G.H. (1967). Electrical impedance and volume changes in brain during preparation for electron microscopy. Brain Res. 5, 57-85.

REynolds, E. S. $(1963)$. The use of lead citrate at high $\mathrm{pH}$ as an electron-opaque stain in electron microscopy. F. Cell Biol. 17, 208-212.

Richardson, K. S., Jarett, L. \& Finke, E. H. (i 960). Embedding in epoxy resins for ultrathin sectioning in electron microscopy. Stain Technol. 35, 313-323.

Van Harreveld, A. \& Crowell, J. (1964). Electron microscopy after rapid freezing on a metal surface and substitution fixation. Anat. Rec. 149, $38_{1-3} 86$.

Van Harreveld, A., Crowell, J. \& Malhotra, S. K. (1965). A study of extracellular space in central nervous tissue by freeze-substitution. $\mathcal{F}$. Cell Biol. 25, i I 7-1 37 .

Van Harreveld, A. \& Khatrab, F. I. (i 968). Perfusion fixation with glutaraldehyde and postfixation with osmium tetroxide for electron microscopy. F. Cell Sci. 3, 579-594.

Van Harreveld, A. \& Khattab, F. I. (I969). Changes in extracellular space of the mouse cerebral cortex during hydroxyadipaldehyde fixation and osmium tetroxide post-fixation. f. Cell Sci. 4, 437-453.

Van Harreveld, A. \& Malhotra, S. K. (1967). Extracellular space in the cerebral cortex of the mouse. F. Anat. 101, 197-207.

VAN Harreveld, A. \& STEINer, J. (1970). Extracellular space in frozen and alcohol substituted central nervous tissue. Anat. Rec. (In the Press.)

(Received 21 $\mathscr{f} u l y$ 1969) 
ABBREVIATIONS ON PLATES

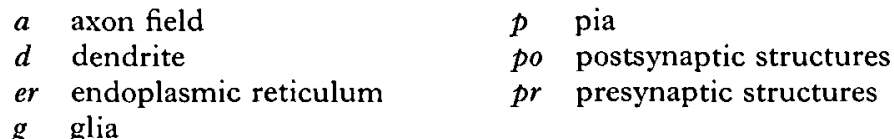

Figs. I, 2. EMs of the surface layer of unexposed cerebral cortex prepared by glutaraldehyde perfusion and $\mathrm{OsO}_{4}$ post-fixation. Note the electron-transparent dendritic structures and the extracellular space in the form of more or less uniform slits around the tissue elements. The scale in Fig. I indicates $1 \mu \mathrm{m}$ and in Fig. 2, $0.2 \mu \mathrm{m}$.

Figs. 3, 4. EMs of a deep region of the unexposed cortex prepared in the same way as the tissue shown in Figs. I, 2. The dendritic structures are more electron-dense than in Fig. $I$ and there is less extracellular space. Some 5 -layered tight junctions are present (arrows). The scale in Fig. 3 indicates $1 \mu \mathrm{m}$, and in Fig. $4,0.2 \mu \mathrm{m}$. 

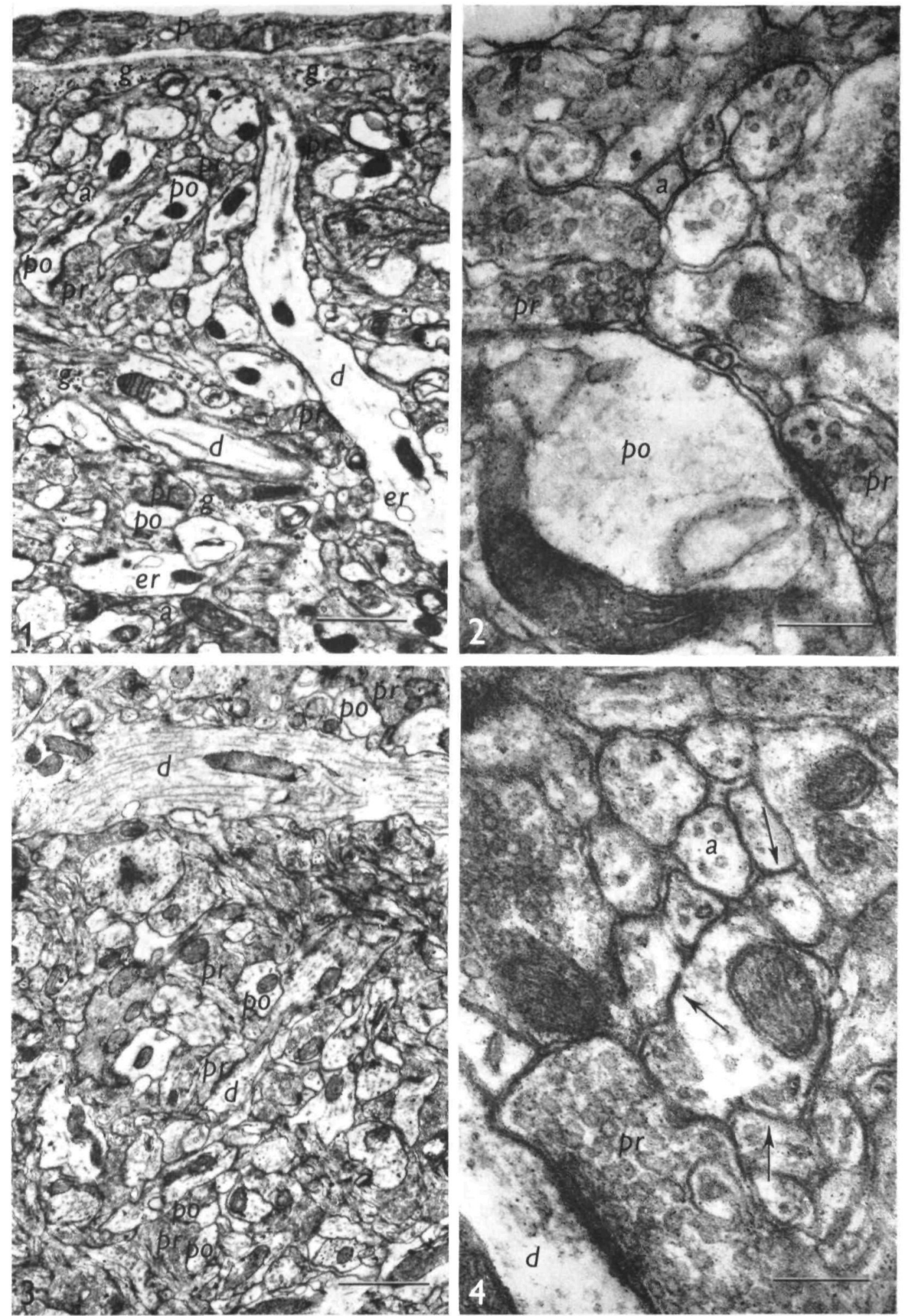
Figs. 5, 6. EMs of the surface layer of the exposed cortex flooded with Ringer's solution while being perfused with the glutaraldehyde fixative, and post-fixed with $\mathrm{OsO}_{4}$. Note the greatly swollen dendritic structures and the wide extracellular slits. The scale in Fig. 5 indicates $1 \mu \mathrm{m}$ and in Fig. $6,0.2 \mu \mathrm{m}$.

Figs. 7,8 . EMs of the surface of the unexposed cortex subjected to freeze substitution after glutaraldehyde perfusion. Note the swollen dendritic structures. There is little extracellular space. At some places 5-layered tight junctions are present between the tissue elements (arrows). The scale in Fig. 7 indicates $1 \mu \mathrm{m}$ and in Fig. $8,0.2 \mu \mathrm{m}$. 


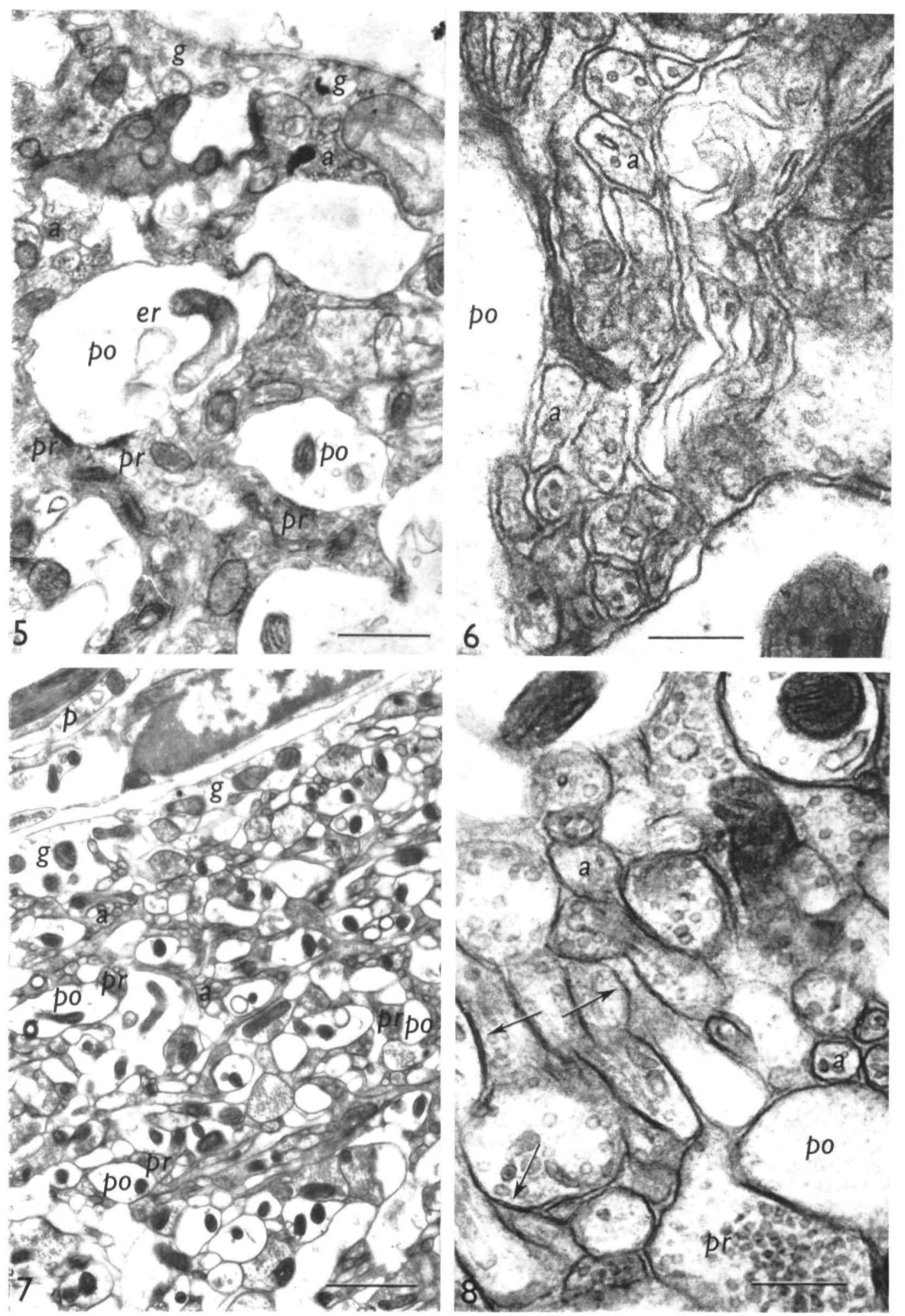


Figs. 9, 10. EMs of the surface of the exposed cortex flooded with Ringer's solution while being perfused with glutaraldehyde and subjected to freeze substitution immediately afterwards. Note the swollen dendritic structures. There is relatively little extracellular space although more than in the EM shown in Fig. 8. Tight junctions are still present (arrows). The scale in Fig. 9 indicates $1 \mu \mathrm{m}$ and in Fig. $10,0.2 \mu \mathrm{m}$.

Figs. I I, I 2. EMs of exposed cortex flooded with Ringer's solution while being perfused with glutaraldehyde, kept for $2 \mathrm{~h}$ in the glutaraldehyde solution and then subjected to freeze substitution. Note the very large and irregular extracellular spaces. The scale in Fig. I I is $\mathrm{I} \mu \mathrm{m}$ and in Fig. $\mathrm{I} 2,0.2 \mu \mathrm{m}$. 

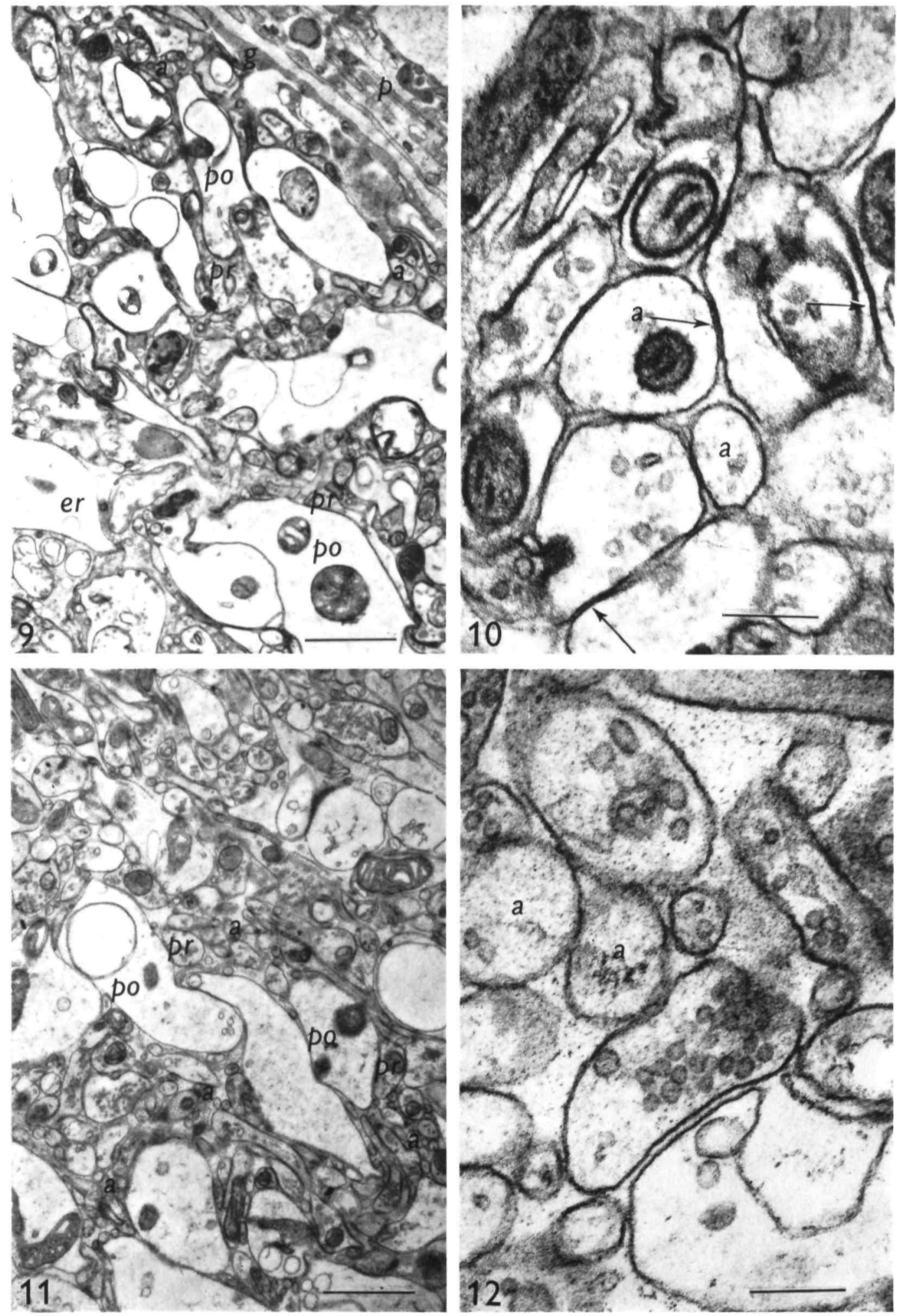
